\title{
Picosecond Dynamics of Non-Thermalized Excited States in Tris(2,2'-bipyridine)ruthenium(II) Derivatives Elucidated by High Energy Excitation
}

\author{
S.A. McFarland, F.S. Lee, K.A.W.Y. Cheng, F.L. Cozens, and N.P. Schepp* \\ Department of Chemistry, Dalhousie University, \\ Halifax, NS B3H 4J3, Canada
}

\section{Supporting Information}

\section{Synthesis}

2,2'-Bipyridine ligands obtained from commercial sources were recrystallized with ethyl acetate to remove impurities prior to ruthenium coordination and were typically isolated as white or pale crystalline solids. Synthesis of the heteroleptic complexes involved preparation and isolation of the $\mathrm{Ru}(\mathrm{LL})_{2} \mathrm{Cl}_{2}$ intermediate from $\mathrm{RuCl}_{3}$ followed by addition of the final bidentate ligand. The tris homoleptic complexes were prepared in a single step directly from $\mathrm{RuCl}_{3}$. Complexes were analyzed by ${ }^{1} \mathrm{H} \mathrm{NMR},{ }^{13} \mathrm{C} \mathrm{NMR}$, and ESI-MS. $\mathrm{Ru}(\text { bpy })_{2} \mathrm{Cl}_{2}$ was purchased from Aldrich while other $\mathrm{Ru}(\mathrm{LL})_{2} \mathrm{Cl}_{2}$ complexes were prepared according to the classical procedure of Sullivan et al. with modifications[1].

4,4'-Di(trifluoromethyl)-2,2'-bypyridine (dfmb) was prepared by the method of Furue et al. [2] with modifications. Activated zinc powder was prepared by washing ground zinc with $0.5 \mathrm{M} \mathrm{HCl}$ followed by water, ethanol, and diethyl ether; the washed zinc was subsequently dried under vacuum at $120^{\circ} \mathrm{C}$ overnight. Tetraethylammonium iodide was dried under vacuum at $110^{\circ} \mathrm{C}$ for $8 \mathrm{~h}$, followed by $16 \mathrm{~h}$ in a vacuum dessicator containing $\mathrm{P}_{2} \mathrm{O}_{5}$. The activated zinc $(1.96 \mathrm{~g}, 30 \mathrm{mmol})$ and tetraethylammonium iodide ( $20 \mathrm{mmol}, 4.0 \mathrm{~g}$ ) were combined with dibromobis(triphenylphosphine)nickel(II) (6 mmol, $4.46 \mathrm{~g}$ ) under argon, to which argon-purged dry THF (70 mL) was added via cannula. After the dark suspension was stirred at room temperature for $30 \mathrm{~min}$, 2-chloro4-(trifluoromethyl)pyridine ( $20 \mathrm{mmol}, 3.6 \mathrm{~g}$, Matrix Scientific) was injected by syringe, and the reaction was stirred at $50^{\circ} \mathrm{C}$ for an additional $48 \mathrm{~h}$ under argon. The black slurry was poured into $2 \mathrm{M}$ ammonium hydroxide $(200 \mathrm{~mL})$, mixed with $200 \mathrm{~mL}$ chloroform, and filtered. The organic layer was separated, dried with anhydrous $\mathrm{Na}_{2} \mathrm{SO}_{4}$, and evaporated in vacuo. Purification of the brown residue by silica gel column chromatography using a gradient eluant of $0.5 \%$ ethyl acetate in hexanes afforded the desired product as a light yellow powder (mp 80-81 ${ }^{\circ} \mathrm{C}$; lit. $\left.81.5-82{ }^{\circ} \mathrm{C}\right)$ in $35 \%$ yield. GC-MS m/z: 292.

5,5'-Di(trifluoromethyl)-2,2'-bypyridine (d5fmb) was synthesized from 2-chloro-5-(trifluoromethyl)pyridine as per the preparation of dfmb. The product was isolated in $24 \%$ yield as a white powder $\left(\mathrm{mp} 109-11{ }^{\circ} \mathrm{C}\right.$; lit. $\left.108.5-110{ }^{\circ} \mathrm{C}\right) .{ }^{1} \mathrm{H} \mathrm{NMR}\left(\mathrm{CDCl}_{3}, 250 \mathrm{MHz}\right) \delta(\mathrm{ppm}): 8.10(\mathrm{~d}, 2 \mathrm{H}, 7.0 \mathrm{~Hz})$, 8.63 (d, 2H, $8.5 \mathrm{~Hz}), 8.96$ (s, 2H). GC-MS m/z: 292.

cis-Bis[4,4'-di(trifluoromethyl)-2,2'-bipyridyl]ruthenium(II) dichloride $\left(\mathbf{R u}(\mathbf{d f m b})_{2} \mathrm{Cl}_{2}\right)$. $\mathrm{RuCl}_{3} \cdot \mathrm{H}_{2} \mathrm{O}$ (1.0 mmol, $250 \mathrm{mg}$, Strem Chemicals) was combined with 4,4'-di(trifluoromethyl)- 
2,2'-bipyridine (2.0 mmol, $864 \mathrm{mg}$ ), flame-dried lithium chloride (6 mmol, $250 \mathrm{mg}$ ), and N,Ndimethylformamide $(5 \mathrm{~mL})$. After refluxing for $8 \mathrm{~h}$ under argon with stirring, the green solution turned blue and was removed from reflux, cooled to room temperature, and quenched with $15 \mathrm{~mL}$ acetone. A purple powder, which appeared after overnight refrigeration, was collected on a fine sintered glass funnel, washed with $2 \times 2 \mathrm{~mL}$ water followed by $3 \times 10 \mathrm{~mL}$ diethyl ether, and dried under vacuum (38\% yield). $\lambda_{a b s}(\mathrm{EtOH}): 388,533 \mathrm{~nm}$.

cis-Bis[5,5'-di(trifluoromethyl)-2,2'-bipyridyl]ruthenium(II) dichloride $\left(\mathbf{R u}(\mathbf{d 5 f m b})_{2} \mathbf{C l}_{2}\right)$. $\mathrm{RuCl}_{3} \cdot \mathrm{H}_{2} \mathrm{O}$ (0.88 mmol, $230 \mathrm{mg}$, Strem Chemicals) was combined with 5,5'-di(trifluoromethyl)2,2'-bipyridine (1.79 mmol, $522 \mathrm{mg}$ ), flame-dried lithium chloride (6 mmol, $250 \mathrm{mg}$ ), and N,Ndimethylformamide $(2 \mathrm{~mL})$ and refluxed for $10 \mathrm{~h}$. The blue solution was removed from reflux, cooled to room temperature, and diluted with $12 \mathrm{~mL}$ acetone. Purple crystals, which appeared after overnight refrigeration, were separated from the blue mother liquor by filtration through a fine sintered glass funnel, washed with $2 \times 1 \mathrm{~mL}$ water followed by $3 \times 5 \mathrm{~mL}$ diethyl ether, and dried under vacuum (67\% yield). $\lambda_{a b s}(\mathrm{EtOH}): 388$ and $533 \mathrm{~nm} .:$ 397, $600 \mathrm{~nm}$.

cis-Bis[4,4'-dimethyl-2,2'-bipyridyl]ruthenium(II) dichloride $\left(\mathbf{R u}(\mathbf{d m b}){ }_{2} \mathbf{C l}_{\mathbf{2}}\right) \cdot \mathrm{RuCl}_{3} \cdot \mathrm{H}_{2} \mathrm{O}(1.0$ mmol, $250 \mathrm{mg}$ ) was combined with 4,4'-dimethyl-2,2'-bipyridine (2.0 mmol, $368 \mathrm{mg}$, Strem Chemicals), flame-dried lithium chloride (6 mmol, $250 \mathrm{mg})$, and N,N-dimethylformamide (5 mL). After refluxing for $8 \mathrm{~h}$ under argon, the brown mixture turned purple and was removed from reflux, cooled to room temperature, and quenched with $20 \mathrm{~mL}$ acetone. A reddish-purple powder, which appeared after overnight refrigeration, was collected on a fine sintered glass funnel, washed with 2 $\times 3 \mathrm{~mL}$ water followed by $5 \times 10 \mathrm{~mL}$ diethyl ether, and dried under vacuum. Suspension of the powder in boiling $100 \mathrm{mM} \mathrm{NaCl}(75 \mathrm{~mL})$ for $10 \mathrm{~min}$ and cooling to $4{ }^{\circ} \mathrm{C}$ [3] gave purple crystals which were collected by filtration and washed with $2 \mathrm{~mL}$ water followed by $10 \mathrm{~mL}$ diethyl ether (12\% yield).

cis-Bis[4,4'-di(methylcarboxy)-2,2'-bipyridyl]ruthenium(II) dichloride ( $\left.\mathbf{R u}(\mathbf{d m e b})_{2} \mathbf{C l}_{2}\right)$. 4,4' Di(methylcarboxy)-2,2'-bipyridine (1.0 mmol, $272 \mathrm{mg}$ ) was combined with anhydrous $\mathrm{RuCl}_{3}(0.5$ mmol, $109 \mathrm{mg}$ ) in $10 \mathrm{~mL}$ methanol and refluxed under argon. After $48 \mathrm{~h}$, the mixture was cooled and dropped into $100 \mathrm{~mL}$ diethyl ether, causing a dark-purple precipitate to form. The precipitate was collected on a fine sintered glass funnel and washed sequentially with $3 \times 20$ mL diethyl ether, $2 \mathrm{~mL}$ ethanol, $3 \times 20 \mathrm{~mL}$ water, $2 \mathrm{~mL}$ ethanol, and $10 \mathrm{~mL}$ diethyl ether. The orange filtrate, presumed to be the tris product, was discarded. The dry purple powder was dissolved in $4 \mathrm{~mL}$ $\mathrm{N}, \mathrm{N}$-dimethylformamide, and the solution was supplemented with $5 \mathrm{~mL}$ acetone and refrigerated overnight. Purple crystals that had formed were collected on a fine sintered glass funnel, washed with $2 \mathrm{~mL}$ water followed by $2 \mathrm{~mL}$ ethanol and $5 \mathrm{~mL}$ diethyl ether, and dried under vacuum (38\% yield)[4]. 
General procedure for the preparation of homoleptic complexes. $\mathrm{RuCl}_{3} \cdot \mathrm{H}_{2} \mathrm{O}(0.01-$ $0.10 \mathrm{mmol}$ ) was combined with $3.5 \mathrm{eq}$. of the appropriate ligand and refluxed in 2-3 mL ethylene glycol under nitrogen until the reaction mixture turned translucent orange (typically 5-30 min, 12-24 h for ligands containing electron-withdrawing trifluoromethyl ligands). After cooling to room temperature, the complexes were precipitated by successive additions of diethyl ether, leaving the dry, crude product adhering to the sides of the flask. Purification was carried out as outlined below.

General procedure for the preparation of heteroleptic complexes. The appropriate ligand (0.01-0.10 mmol) was combined with 0.80 eq. of the desired cis-bis(bipyridyl)Ru(II) dichloride and refluxed in 5:1 methanol:acetic acid[5] (10-25 mL) under argon. After the mixture turned translucent orange (typically $10 \mathrm{~min}-6 \mathrm{~h}$ ), the reaction was cooled and evaporated in vacuo to yield the crude product. Purification was carried out as outlined below.

General procedure for purification by precipitation[6]. The crude product $(>0.02 \mathrm{mmol})$ was dissolved in 1-5 mL acetonitrile, slowly dropped into $100 \mathrm{~mL}$ anhydrous diethyl ether to remove any remaining ligand, and collected on a fine sintered glass funnel. The contents of the funnel were dissolved in a minimum amount of doubly distilled water $(1-4 \mathrm{~mL})$ and gently drawn through the filter by suction to remove water-insoluble components. In the case of $\mathrm{Ru}(\mathrm{bpy})_{2} \mathrm{Cl}_{2}$, the orange aqueous solution was combined with 5-10 mL acetonitrile or methanol and evaporated in vacuo; addition of cosolvents was usually necessary to facilitate the removal of water. Typical isolated yields were 60-90\%.

General procedure for purification by size-exclusion chromatography[2,5]. The crude product $(<0.02 \mathrm{mmol}$ ) was dissolved in $0.5 \mathrm{~mL}$ methanol, loaded onto a Sephadex LH-20 column $(30 \times 3 \mathrm{~cm}$ diameter), and eluted with methanol at a flow rate of $c a .2 \mathrm{~mL} / \mathrm{min}$. The first coloured band (orange; $5-10 \mathrm{~mL}$ ) to elute was collected and evaporated in vacuo to afford the product (80$90 \%)$.

Tris(2,2'-bipyridyl-d8)ruthenium(II) dichloride ( $\left.\mathbf{R u}(\mathbf{p d b})_{3} \mathbf{C l}_{2}\right)(\mathbf{1})$. Prepared from $\mathrm{RuCl}_{3} \cdot 3 \mathrm{H}_{2} \mathrm{O}$ and perdeuterated 2,2'-bipyridine (99.8\% atom D, CDN Isotopes). ${ }^{1} \mathrm{H}$ NMR $\left(\mathrm{CD}_{3} \mathrm{OD}, 250 \mathrm{MHz}\right)$ $\delta$ (ppm): 7.52 (s), 7.84 (s), 8.18 (s), 8.77 (s). ESI-MS m/z: 297.3 (100\%).

Tris(4,4'-dimethyl-2,2'-bipyridyl)ruthenium(II) dichloride $\left(\mathrm{Ru}(\mathrm{dmb})_{3} \mathrm{Cl}_{2}\right)(2)$. Prepared from $\mathrm{RuCl}_{3} \cdot 3 \mathrm{H}_{2} \mathrm{O}$ and 4,4'-dimethyl-2,2'-bipyridine. ${ }^{1} \mathrm{H} \mathrm{NMR}\left(\mathrm{CD}_{3} \mathrm{OD}, 250 \mathrm{MHz}\right) \delta(\mathrm{ppm}): 2.55(\mathrm{~s}$, 18H), 7.30 (d, 6H, $5.8 \mathrm{~Hz}$ ), 7.57 (d, 6H, $5.8 \mathrm{~Hz}$ ), 8.58 (s, 6H). ESI-MS m/z: 327.3 (100\%).

Tris(5,5'-dimethyl-2,2'-bipyridyl)ruthenium(II) dichloride $\left(\mathrm{Ru}(\mathrm{d5mb})_{3} \mathrm{Cl}_{2}\right)(3)$. Prepared from $\mathrm{RuCl}_{3} \cdot 3 \mathrm{H}_{2} \mathrm{O}$ and 5,5'-dimethyl-2,2'-bipyridine. ${ }^{1} \mathrm{H} \mathrm{NMR}\left(\mathrm{CD}_{3} \mathrm{OD}, 250 \mathrm{MHz}\right) \delta(\mathrm{ppm}): 2.25(\mathrm{~s}$, 18H), 7.55 (s, 6H), 7.94 (d, 6H, 7.9 Hz), 8.53 (d, 6H, 8.1 Hz). ESI-MS m/z: 327.2 (100\%).

Tris(4,4'-dimethoxy-2,2'-bipyridyl)ruthenium(II) dichloride ( $\left.\mathrm{Ru}(\mathbf{d m e o b})_{3} \mathrm{Cl}_{2}\right)$ (4). Prepared from $\mathrm{RuCl}_{3} \cdot 3 \mathrm{H}_{2} \mathrm{O}$ and 4,4'-dimethoxy-2,2'-bipyridine. ${ }^{1} \mathrm{H} \mathrm{NMR}\left(\mathrm{CD}_{3} \mathrm{OD}, 250 \mathrm{MHz}\right) \delta(\mathrm{ppm})$ : $4.03(\mathrm{~s}, 18 \mathrm{H}), 7.06$ (d, 6H, $6.7 \mathrm{~Hz}), 7.60$ (d, 6H, 6.7 Hz), 8.26 (s, 6H). ESI-MS m/z: $375.2(100 \%)$ and $750.0(5 \%)$. 
Tris[4,4'-di(trifluoromethyl)-2,2'-bipyridyl]ruthenium(II) dichloride ( $\left.\mathrm{Ru}(\mathrm{dfmb})_{3} \mathrm{Cl}_{2}\right)(5)$. Prepared from $\mathrm{RuCl}_{3} \cdot 3 \mathrm{H}_{2} \mathrm{O}$ and 4,4'-di(trifluoromethyl)-2,2'-bipyridine. ${ }^{1} \mathrm{H}$ NMR $\left(\mathrm{CD}_{3} \mathrm{OD}, 250\right.$ MHz) $\delta(\mathrm{ppm}): 7.85$ (d, 6H, $5.2 \mathrm{~Hz}, 8.16$ (d, 6H, $5.8 \mathrm{~Hz}), 9.38$ (s, 6H). ESI-MS m/z: 489.0 (100\%), $977.0(15 \%)$.

Bis(2,2'-bipyridyl)(4,4'-dimethyl-2,2'-bipyridyl)ruthenium(II) dichloride ( $\left.\mathbf{R u}(\mathbf{b p y})_{2}(\mathbf{d m b}) \mathrm{Cl}_{2}\right)$ (6). Prepared from $\mathrm{Ru}(\mathrm{bpy})_{2} \mathrm{Cl}_{2}$ and 4,4'-dimethyl-2,2' -bipyridine. ${ }^{1} \mathrm{H} \mathrm{NMR}\left(\mathrm{CD}_{3} \mathrm{OD}, 250 \mathrm{MHz}\right)$ $\delta(\mathrm{ppm}): 2.56(\mathrm{~s}, 6 \mathrm{H}), 7.29(\mathrm{~d}, 2 \mathrm{H}, 5.2 \mathrm{~Hz}), 7.48(\mathrm{~m}, 4 \mathrm{H}), 7.58(\mathrm{~d}, 2 \mathrm{H}, 5.8 \mathrm{~Hz}), 7.79(\mathrm{~m}, 4 \mathrm{H}), 8.09$ (t, 4H, $7.8 \mathrm{~Hz}), 8.58$ (s, 2H), 8.67 (d, 4H, 8.2 Hz). ESI-MS m/z: 299.3 (100\%).

Bis(2,2'-bipyridyl)(5,5'-dimethyl-2,2'-bipyridyl)ruthenium(II) dichloride ( $\left.\operatorname{Ru}(\mathbf{b p y})_{2}(\mathbf{d} 5 \mathrm{mb}) \mathbf{C l}_{2}\right)$ (7). Prepared from $\mathrm{Ru}(\mathrm{bpy})_{2} \mathrm{Cl}_{2}$ and 5,5'-dimethyl-2,2'-bipyridine. ${ }^{1} \mathrm{H}$ NMR $\left(\mathrm{CD}_{3} \mathrm{OD}, 250 \mathrm{MHz}\right)$ $\delta(\mathrm{ppm}): 2.26(\mathrm{~s}, 6 \mathrm{H}), 7.53(\mathrm{~m}, 6 \mathrm{H}), 7.83(\mathrm{~d}, 4 \mathrm{H}, 5.5 \mathrm{~Hz}), 7.96(\mathrm{~d}, 2 \mathrm{H}, 8.6 \mathrm{~Hz}), 8.15(\mathrm{t}, 4 \mathrm{H}, 7.6$ Hz), 8.57 (d, 2H, 8.2 Hz), 8.75 (d, 4H, 7.9 Hz). ESI-MS m/z: 299.2 (100\%), 597.1 (20\%), 632.8 $(10 \%)$.

\section{Bis(2,2'-bipyridyl)(4,4'-dimethoxy-2,2'-bipyridyl)ruthenium(II) dichloride}

(Ru(bpy) $\left.)_{2}(\mathbf{d m e o b}) \mathbf{C l}_{2}\right)$ (8). Prepared from $\mathrm{Ru}(\text { bpy })_{2} \mathrm{Cl}_{2}$ and $4,4^{\prime}$-dimethoxy-2,2'-bipyridine. ${ }^{1} \mathrm{H} \mathrm{NMR}$ $\left(\mathrm{CD}_{3} \mathrm{OD}, 250 \mathrm{MHz}\right) \delta(\mathrm{ppm}): 2.57(\mathrm{~s}, 6 \mathrm{H}), 2.59(\mathrm{~s}, 6 \mathrm{H}), 4.04(\mathrm{~s}, 6 \mathrm{H}), 7.06(\mathrm{~m}, 2 \mathrm{H}), 7.29(\mathrm{~d}, 2 \mathrm{H}$, $5.8 \mathrm{~Hz}), 7.33$ (d, 2H, $5.8 \mathrm{~Hz}), 7.53(\mathrm{~d}, 2 \mathrm{H}, 6.4 \mathrm{~Hz}), 7.60$ (d, 2H, $5.8 \mathrm{~Hz}), 7.70$ (d, 2H, $5.5 \mathrm{~Hz}), 8.28$ (d, 2H, $2.8 \mathrm{~Hz}), 8.56$ (s, 4H). ESI-MS m/z: 685.1 (100\%) and $343.2(20 \%)$.

Bis(2,2'-bipyridyl)(4,4'-di(trifluoromethyl)-2,2'-bipyridyl]ruthenium(II) dichloride $\left(\mathbf{R u}(\mathbf{b p y})_{2}(\mathbf{d f m b}) \mathbf{C l}_{2}\right)(\mathbf{9})$. Prepared from $\mathrm{Ru}(\mathrm{bpy})_{2} \mathrm{Cl}_{2}$ and 4,4'-di(trifluoromethyl)-2,2'-bipyridine. ${ }^{1} \mathrm{H} \mathrm{NMR}\left(\mathrm{CD}_{3} \mathrm{OD}, 250 \mathrm{MHz}\right) \delta(\mathrm{ppm}): 7.56(\mathrm{~m}, 4 \mathrm{H}), 7.85(\mathrm{~m}, 6 \mathrm{H}), 8.19(\mathrm{~m}, 6 \mathrm{H}), 8.80(\mathrm{~d}, 4 \mathrm{H}, 7.5$ $\mathrm{Hz}), 9.32$ (s, 2H). ESI-MS m/z: 353.3 (100\%).

Bis(2,2'-bipyridyl)[5,5'-di(trifluoromethyl)-2,2'-bipyridyl]ruthenium(II) dichloride $\left(\mathbf{R u}(\mathbf{b p y})_{2}(\mathbf{d 5 f m b}) \mathbf{C l}_{2}\right) \mathbf{( 1 0 )}$. Prepared from $\mathrm{Ru}(\mathrm{bpy})_{2} \mathrm{Cl}_{2 \mathrm{j}}$ and 5,5'-di(trifluoromethyl)-2,2'-bipyridine. ${ }^{1} \mathrm{H}$ NMR $\left(\mathrm{CD}_{3} \mathrm{OD}, 250 \mathrm{MHz}\right) \delta(\mathrm{ppm}): 7.55(\mathrm{~m}, 4 \mathrm{H}), 7.84(\mathrm{~d}, 2 \mathrm{H}, 4.9 \mathrm{~Hz}), 7.91(\mathrm{~d}, 2 \mathrm{H}, 4.9 \mathrm{~Hz})$, $7.98(\mathrm{~s}, 2 \mathrm{H}), 8.20(\mathrm{~m}, 4 \mathrm{H}), 8.24(\mathrm{t}, 2 \mathrm{H}, 8.3 \mathrm{~Hz}), 8.53(\mathrm{~d}, 2 \mathrm{H}, 8.9 \mathrm{~Hz}), 8.77(2 \mathrm{~d}, 4 \mathrm{H}), 9.12(\mathrm{~d}, 2 \mathrm{H}$, $8.5 \mathrm{~Hz})$. ESI-MS m/z: $353.1(100 \%)$.

Bis[4,4'-di(trifluoromethyl)-2,2'-bipyridyl](2,2'-bipyridyl)ruthenium(II) dichloride (Ru(dfmb $\left.)_{\mathbf{2}}(\mathbf{b p y}) \mathbf{C l}_{2}\right)$ (11). Prepared from $\mathrm{Ru}(\mathrm{dfmb})_{2} \mathrm{Cl}_{2}$ and 2,2'-bipyridine. ${ }^{1} \mathrm{H}$ NMR $\left(\mathrm{CD}_{3} \mathrm{OD}\right.$, $250 \mathrm{MHz}) \delta(\mathrm{ppm}): 7.56(2 \mathrm{~d}, 2 \mathrm{H}), 7.85(\mathrm{~m}, 6 \mathrm{H}), 8.13-8.25(\mathrm{~m}, 6 \mathrm{H}), 8.78(\mathrm{~d}, 2 \mathrm{H}, 7.5 \mathrm{~Hz}), 9.35$ (s, 4H). ESI-MS m/z: $421.2(100 \%), 876.5$ (7\%).

Bis[5,5'-di(trifluoromethyl)-2,2'-bipyridyl](2,2'-bipyridyl)ruthenium(II) dichloride $\left(\mathbf{R u}(\mathbf{d 5 f m b})_{\mathbf{2}}(\mathbf{b p y}) \mathbf{C l}_{2}\right)$ (12). Prepared from $\mathrm{Ru}(\mathrm{d} 5 \mathrm{fmb})_{2} \mathrm{Cl}_{2}$ and 2, $2^{\prime}$-bipyridine. ${ }^{1} \mathrm{H} \mathrm{NMR}\left(\mathrm{CD}_{3} \mathrm{OD}\right.$, $250 \mathrm{MHz}) \delta(\mathrm{ppm}): 7.59(\mathrm{t}, 2 \mathrm{H}, 6.1 \mathrm{~Hz}), 7.88(\mathrm{~d}, 2 \mathrm{H}, 5.5 \mathrm{~Hz}), 7.96(\mathrm{~s}, 2 \mathrm{H}), 8.17(\mathrm{~s}, 2 \mathrm{H}), 8.24(\mathrm{t}$, 2H, $8.3 \mathrm{~Hz}), 8.60(2 \mathrm{~d}, 4 \mathrm{H}), 8.79$ (d, 2H, $8.0 \mathrm{~Hz}), 9.12$ (d, 4H, 8.6 Hz). ESI-MS m/z: $421.2(100 \%)$.

Bis[4,4'-di(trifluoromethyl)-2,2'-bipyridyl](4,4'-dimethyl-2,2'-bipyridyl)-ruthenium(II) dichloride $\left(\mathbf{R u}(\mathbf{d f m b})_{2}(\mathbf{d m b}) \mathbf{C l}_{2}\right)(\mathbf{1 3})$. Prepared from $\mathrm{Ru}(\mathrm{dfmb})_{2} \mathrm{Cl}_{2}$ and 4,4'-dimethyl-2,2'-bipyridine. ${ }^{1} \mathrm{H} \mathrm{NMR}\left(\mathrm{CD}_{3} \mathrm{OD}, 250 \mathrm{MHz}\right) \delta(\mathrm{ppm}): 2.59(\mathrm{~s}, 12 \mathrm{H}), 7.34(2 \mathrm{~d}, 4 \mathrm{H}), 7.60(2 \mathrm{~d}, 4 \mathrm{H}), 7.79(\mathrm{~d}, 2 \mathrm{H}$, $4.9 \mathrm{~Hz}), 8.15$ (d, 2H, $5.8 \mathrm{~Hz}), 8.60$ (s, 4H), 9.28 (s, 2H). ESI-MS m/z: 381.1 (70\%), 761.0 (100\%). 
Bis[5,5'-di(trifluoromethyl)-2,2'-bipyridyl](5,5'-dimethyl-2,2'-bipyridyl)-ruthenium(II) dichloride ( $\left.\mathbf{R u}(\mathbf{d 5 f m b})_{\mathbf{2}}(\mathbf{d 5 m b}) \mathbf{C l}_{2}\right)$ (14). Prepared from $\mathrm{Ru}(\mathrm{d} 5 \mathrm{fmb})_{2} \mathrm{Cl}_{2}$ and 5,5'-dimethyl-2,2' -bipyridine. ${ }^{1} \mathrm{H} \mathrm{NMR}\left(\mathrm{CD}_{3} \mathrm{OD}, 250 \mathrm{MHz}\right) \delta(\mathrm{ppm}): 2.29(\mathrm{~s}, 6 \mathrm{H}), 7.68(\mathrm{~s}, 2 \mathrm{H}), 7.95(\mathrm{~s}, 2 \mathrm{H}), 8.04$ (d, 2H, 7.6 Hz), 8.15 (s, 2H), 8.61 (m, 6H), 9.13 (m, 4H). ESI-MS m/z: 435.1 (100\%), 597.1 (20\%), 868.9 $(22 \%)$.

Bis[4,4'-di(trifluoromethyl)-2,2'-bipyridyl](4,4'-dimethoxy-2,2'-bipyridyl)-ruthenium(II) dichloride $\left(\mathbf{R u}(\mathbf{d f m b})_{\mathbf{2}}(\mathbf{d m e o b}) \mathbf{C l}_{\mathbf{2}}\right)$ (15). Prepared from $\mathrm{Ru}(\mathrm{dfmb})_{2} \mathrm{Cl}_{2}$ and 4,4'-dimethoxy-2,2'-bipyridine. ${ }^{1} \mathrm{H}$ NMR $\left(\mathrm{CD}_{3} \mathrm{OD}, 250 \mathrm{MHz}\right) \delta(\mathrm{ppm}): 4.05(\mathrm{~s}, 6 \mathrm{H}), 7.09(\mathrm{~d}, 1 \mathrm{H}, 2.8 \mathrm{~Hz}), 7.11(\mathrm{~d}, 1 \mathrm{H}, 2.8 \mathrm{~Hz})$, $7.53(\mathrm{~d}, 2 \mathrm{H}, 6.7 \mathrm{~Hz}), 7.78(\mathrm{~d}, 2 \mathrm{H}, 5.8 \mathrm{~Hz}), 7.88(\mathrm{~d}, 2 \mathrm{H}, 5.9 \mathrm{~Hz}), 8.16(\mathrm{~d}, 2 \mathrm{H}, 6.1 \mathrm{~Hz}), 8.21(\mathrm{~d}, 2 \mathrm{H}$, $5.8 \mathrm{~Hz}$, 8.34 (d, 2H, 2.8 Hz), 9.33 (s, 4H). ESI-MS m/z: 451.1 (100\%), 936.5 (10\%). 


\section{Figures}

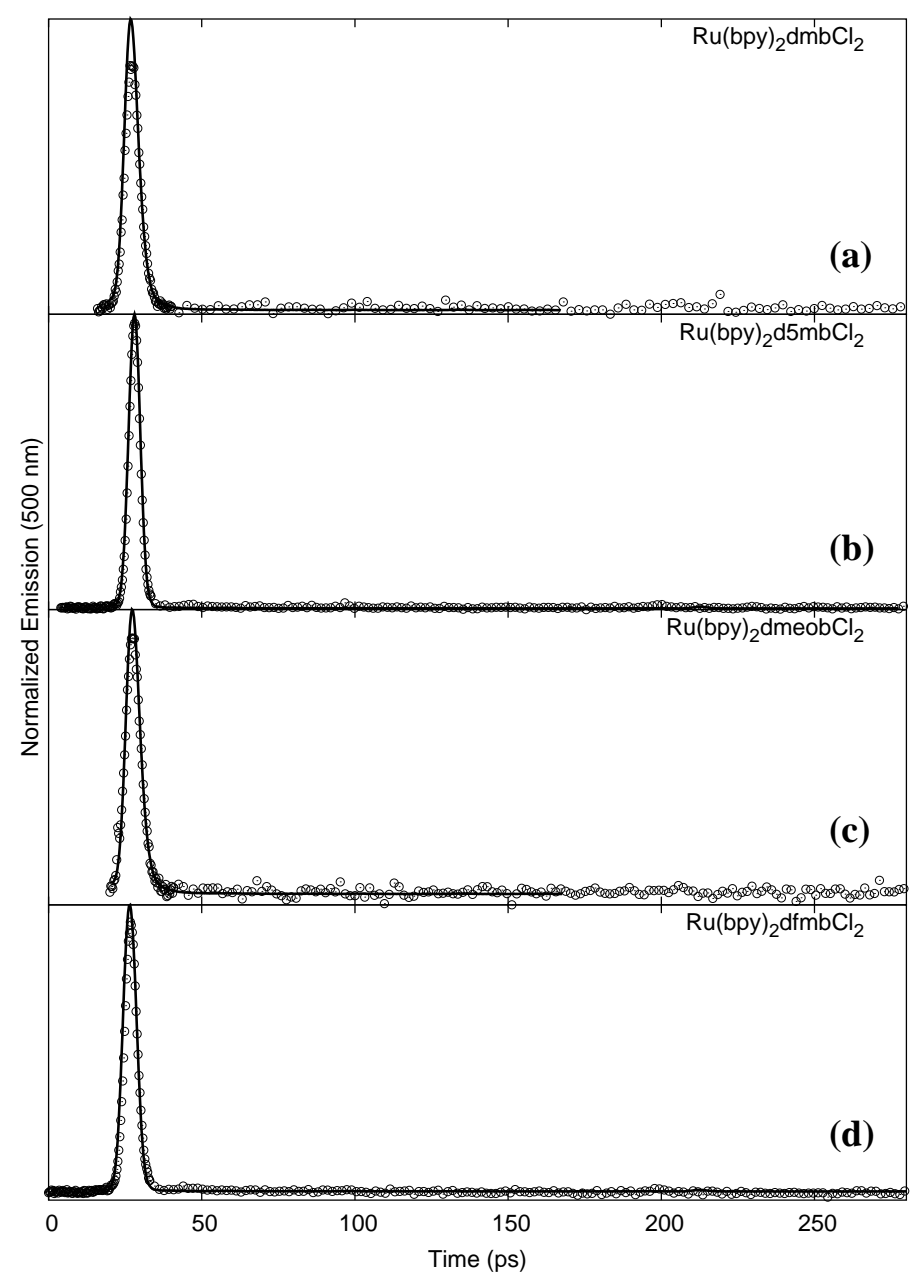

Figure S1: Luminescence signal of $\mathrm{Ru}(\mathrm{bpy})_{2}(\mathrm{dmb})^{2+}(\mathrm{a}), \quad \mathrm{Ru}(\mathrm{bpy})_{2}(\mathrm{~d} 5 \mathrm{mb})^{2+}(\mathrm{b})$, $\mathrm{Ru}(\mathrm{bpy})_{2}(\mathrm{dmeob})^{2+}(\mathrm{c})$, and $\mathrm{Ru}(\mathrm{bpy})_{2}(\mathrm{dfmb})^{2+}(\mathrm{d})$ in $\mathrm{H}_{2} \mathrm{O}$ at $500 \mathrm{~nm}\left(\lambda_{e x}=388 \mathrm{~nm}\right)$. Open circles represent the actual data, and solid lines represent the instrument response function (IRF). 


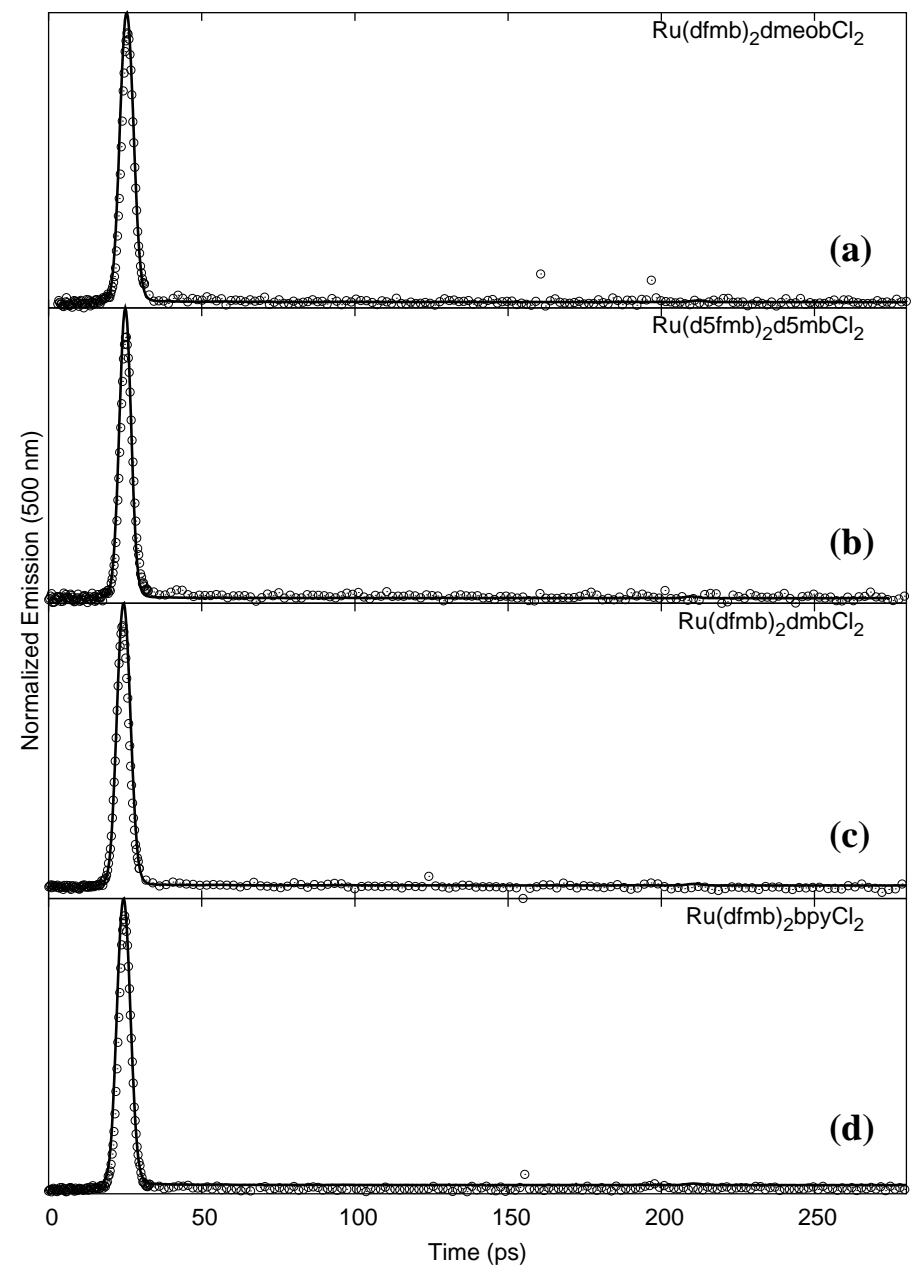

Figure S2: Luminescence signal of $\mathrm{Ru}(\mathrm{dfmb})_{2}(\mathrm{dmeob})^{2+}(\mathrm{a}), \quad \mathrm{Ru}(\mathrm{d} 5 \mathrm{fmb})_{2}(\mathrm{~d} 5 \mathrm{mb})^{2+}(\mathrm{b})$, $\mathrm{Ru}(\mathrm{dfmb})_{2}(\mathrm{dmb})^{2+}(\mathrm{c})$, and $\mathrm{Ru}(\mathrm{dfmb})_{2}(\mathrm{bpy})^{2+}(\mathrm{d})$ in $\mathrm{H}_{2} \mathrm{O}$ at $500 \mathrm{~nm}\left(\lambda_{e x}=388 \mathrm{~nm}\right)$. Open circles represent the actual data, and solid lines represent the instrument response function (IRF). 


\section{References}

[1] Sullivan, B.; Salmon, D.; Meyer, T. Inorg. Chem. 1978, 17, 3334-3341.

[2] Furue, M.; Maruyama, K.; Oguni, T.; Naiki, M.; Kamachi, M. Inorg. Chem. 1992, 31, 3792-3795.

[3] Yang, J.; Seneviratne, D.; Arbatin, G.; Anderson, A.; Curtis, J. J. Am. Chem. Soc. 1997, 119, 5329-5336.

[4] Hou, Y.; Xie, P.; Zhang, B.; Cao, Y.; Xiao, X.; Wang, W. Inorg. Chem. 1999, 38, 63206322.

[5] Hesek, D.; Inoue, Y.; Everitt, S.; Ishida, H.; Kunieda, M.; Drew, M. Inorg. Chem. 2000, 39, 308-316.

[6] Torki, F. E.; Schmehl, R.; Reed, W. J. Chem. Soc. Faraday Trans. 1 1989, 85, 349-362. 